NO.10 Journal of Petroleum Research \& Studies ( JPR \& S)

\title{
Adoption the basis of spatial analysis and geographic information systems (GIS) techniques to development oil industry projects
}

\author{
Fanir Farooq Omar Naji
}

North Oil Company

\section{Submitted}

It is recognized today that human lives the information age, which is characterized by the multiplicity of its data and the complexity of its aspects and the most important thing is characterized by "information" that will determine the progress of the people, the more able countries to devise ways to protect the information and how to make the best use of them whenever it is an indication of its progress and its ability to face obstacles to development and the information sources are diverse and interrelated up to the level of complexity that they can be analyzed and dealt with the existence of developed computer systems, that stored and processed the information. And because maps are also considered sources of information which use in management and project planning, and being an important means of connecting ideas and planning them so it necessitated the need for the emergence of advanced systems have the ability to draw and design schemes and production of digital maps. With possibility of linking information (metadata) with the real location on the Earth's surface, called the (spatial data). The emergence of these systems are scientific leap on the in geographic science especially spatial data that has accumulated over the centuries and resulted in a huge amount of data and information. These systems added a lot in the field of building spatial database which can updating and help in treating maps accurately and effectively combines the advantages of the two systems (information systems and systems of digital maps), which was named "GIS" systems which electronic 


\section{NO.10 Journal of Petroleum Research \& Studies ( JPR \& S)}

digital competent to enter, store and retrieve, analyze data . And connect them with large areas of maps that can not be saved on security on the paper. The data is saved with maps in a coherent manner so as to facilitate the user to display the data with maps and several methods as well as a calculation processing to extract the results and reports in a few time and effort and use them in decision-making which helps to speed the study and analysis the information and support decision-makers in a number of important decisions concerning projects which requires accuracy and speed.

\section{Objective of the research}

This research aims to publicize the importance of geographic information systems and the necessity to employ the system in the institutions and use it for build a geographic database of areas that fall within the responsibilities of these institutions and used mainly to benefit from their high ability in the documentation and analysis and other capabilities required by the nature of the work in these institutions that deal with large amounts of data and information and convert fixed maps to the animated maps, with the ability of moving, deletions and added without the need to return work again as is the case with conventional methods. As it can be linked the geographical databases with basis maps and build several layers of geographic information that can be used in the analysis and, application, and allows an easy opportunity to analyze and treatment a wide range of data to support strategic decision that based on a logical choice for a perfect choice among several choices. And to maximize the use of this data it converted to the form that enables more than one user or management access with it and works on linking many of the databases that follows each of them to a certain destination, at the same time each side update their databases permanently, that create a spatial database integrated and updated with the number of layers of information that used 


\section{NO.10 Journal of Petroleum Research \& Studies ( JPR \& S)}

in different applications, and saves a lot of time, effort and cost and contribute to the preparation of the required analysis to develop the productivity.

\section{Study area}

The study included sites from the residential Baba area and $\mathrm{k} 1$ area which include $\mathrm{k} 1$ tanks and pipes extended to drain and receipt of oil between the reservoirs and valves that control the flow of the oil in the pipe. as well as other features such as buildings and departments of the North Oil Company and the streets which appear in the image space. The aerial photographs and satellite imagery and paper maps are the important sources of geographic data. Therefore, when institutions establish a INCLUSIVE geographic information system so the users of this data from different departments can reduce the time required to perform the tasks required on spatial data and integration the data to implement the tasks. It could be argued that the geographic information systems consist of technologies and human competencies and different data sources that needed to create and save and show the geographic data and leading to the establishment of an effective system that supports spatial decision-making

\section{Practical applications of geographic information systems in petroleum baba area}

The application of the system in a specific area begins with provide satellite image of the area and collect data and information from various sources such as reports, paper maps and other sources of information to represent spatial database and information required to complete the construction of geographic database. The paper maps are spatial data that can be beneficial after scanning and correcting it geographicaly and engineering and converted it to a mechanism form. Where that integration between paper maps and satellite image lead to create an integrated spatial information system for required area. 
NO.10 Journal of Petroleum Research \& Studies ( JPR \& S)

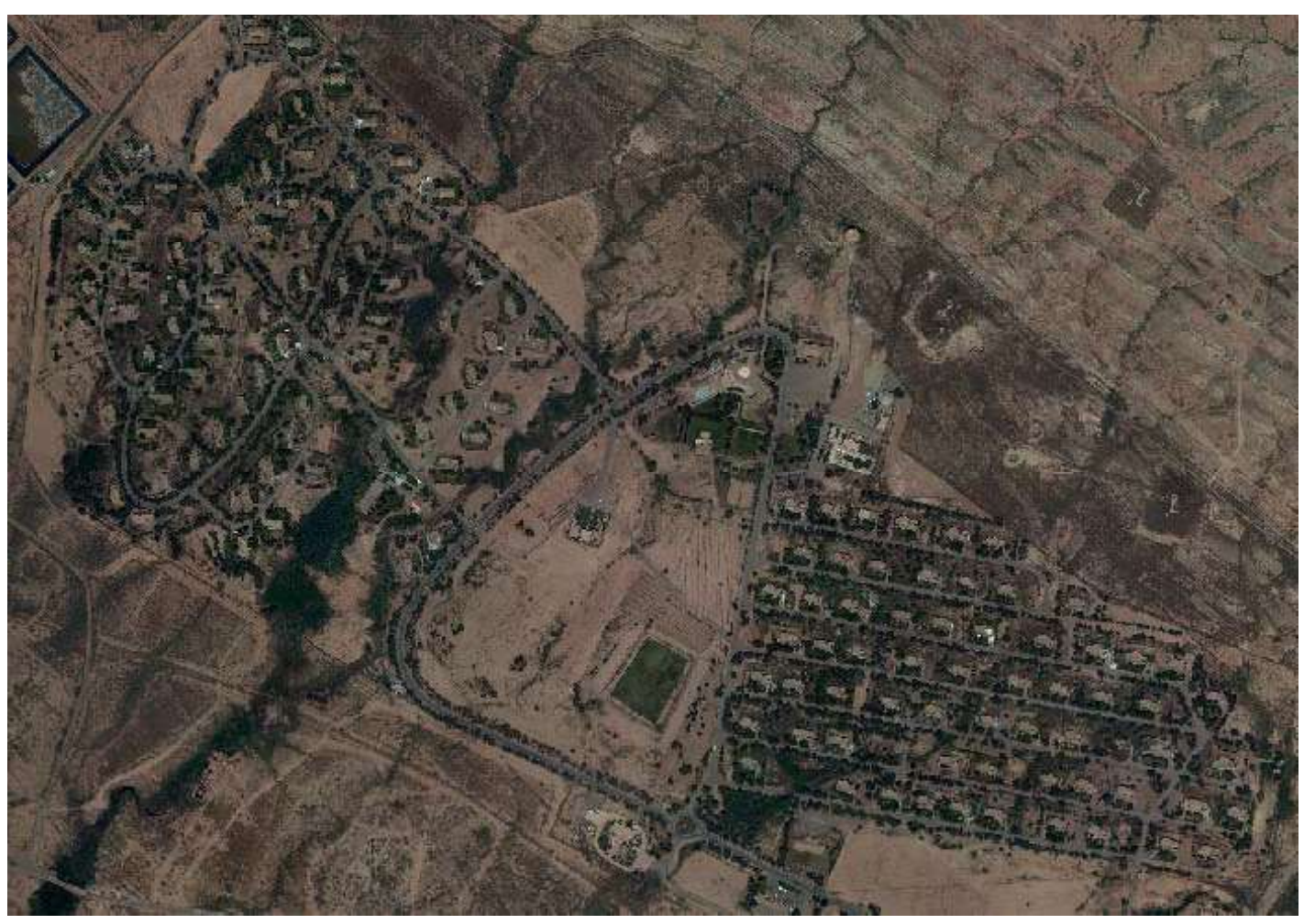

Fig. (1) Model of satellite image for a residential area in Baba area

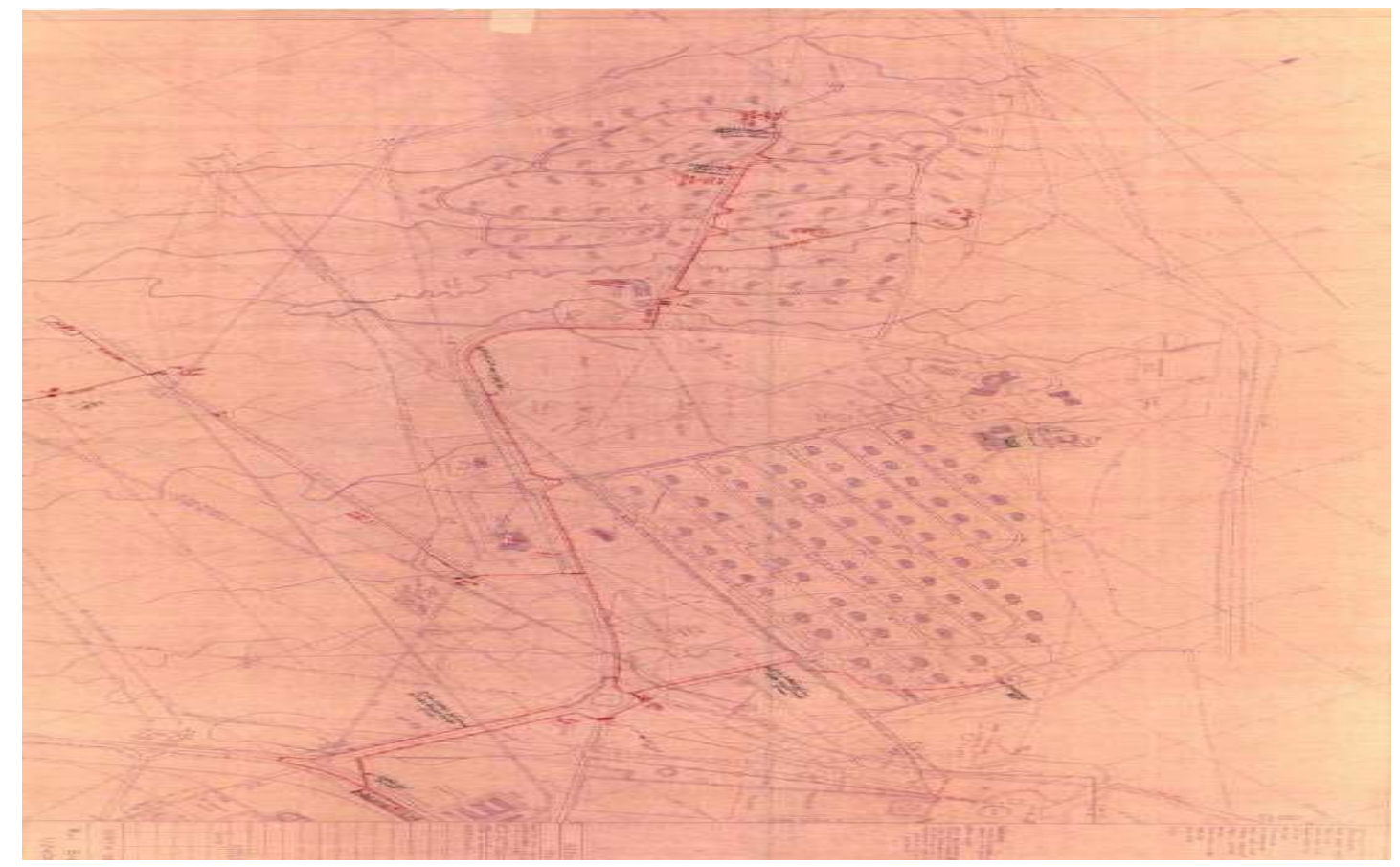

Fig. (2) Paper map of the area after correct it by realistic point 


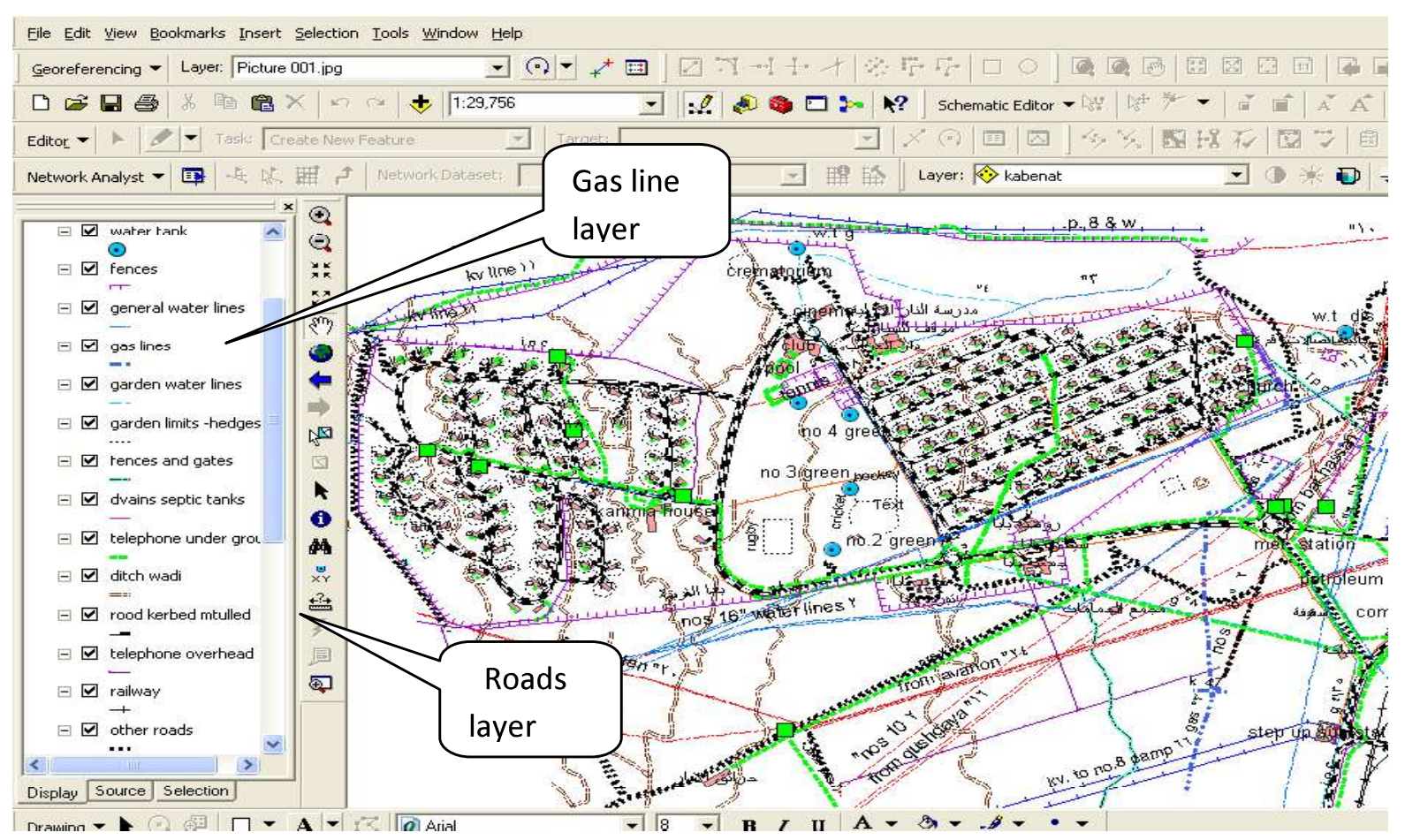

Fig. (3)

Figure (3) shows screen of the system whereas the map has been drawing by dependence on satellite image and paper map, so the geographic information systems is not only to display maps on the computer screen, but they are interactive, intelligent systems, contain a huge amount of information that is working on store and call and analysis of each of the geographical and descriptive data easily, and displayed them on a computer screen or on paper in the form of maps, reports, and graphs with the required information and make statistical comparisons. 


\section{NO.10}

Journal of Petroleum Research \& Studies ( JPR \& S)

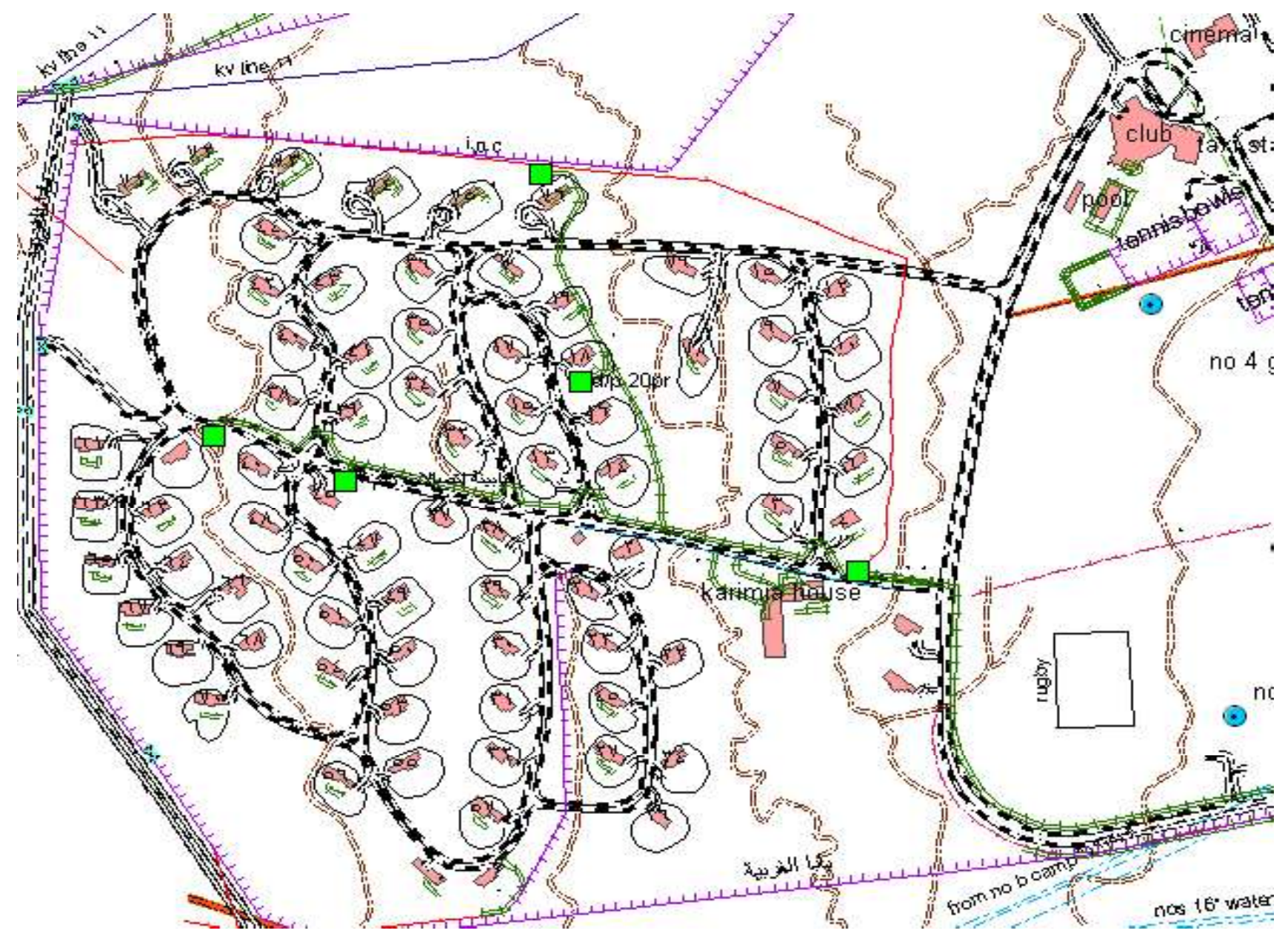

Fig. (4)

Figure (4) appears enlarged part of the map, which represents the Eastern Baba are so one of the functions provided by geographic information systems creates a variety of maps depending on the required task. 


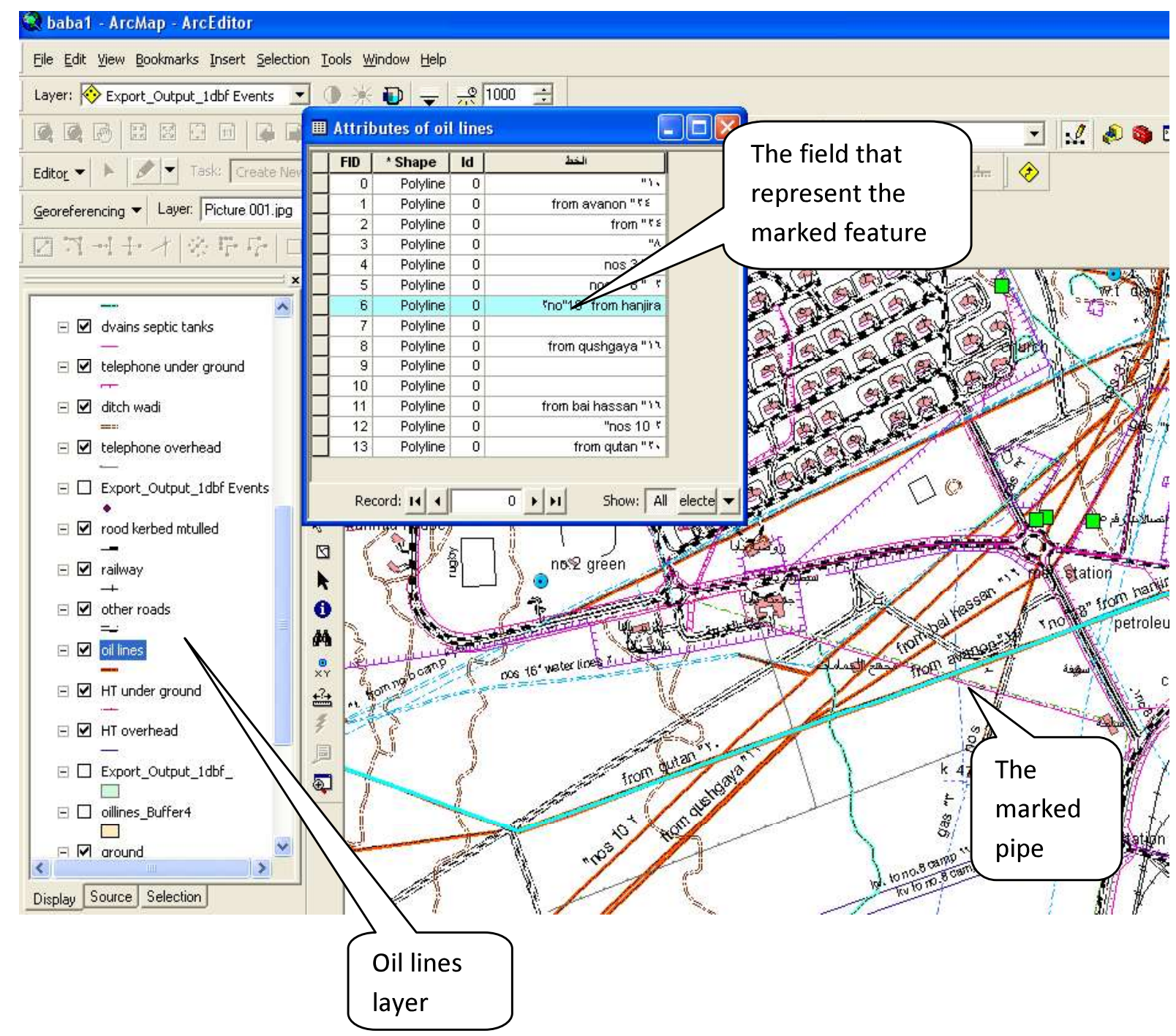

Fig. (5)

Figure (5) shows one of the characteristics of the system which is access easiness to information about phenomenon features on the map, such as when opening the information table of oil pipe layer. And choose any field which represent any oil pipeline so it will be marked in the map also when we choose it on the map we can see its field marked 
NO.10 Journal of Petroleum Research \& Studies ( JPR \& S)

Employment one of the spatial analysis techniques in geographic information system with an explanation of the importance of this technology in identifying the areas that need protection

One of the distinct functions in GIS system is known by spatial analysis systems which based on a clear methodology in modeling spatial information in order to identify the most suitable sites where as achieve the highest level of the pros and minimize the damage. And that based on a set of standard information which is converted digitally in geographic information systems.

Since the system's ability to integrate information from multiple sources in the spatial frame and comprehensive analysis of several concepts related to measurement and classification, arrangement and corresponding and geographic surface analysis and other concepts that can be combined and lead to access to the correct choice and find the appropriate place to accomplish a specific goal available map represent the residential area in Baba area, as well as government buildings in the region with design for oil and gas lines and telephone cables lines and communications cabin and other features appear in the map. The required is to identify areas susceptible to risk more than other, which are closer to the oil pipes or oil terminals and these areas include the streets and buildings, so the system can choice these features and isolated them in a special table to take security measures in these areas more than others. The process of analysis and knowledge of the area include an event depend on measuring a specified distance from the feature, which in this case is the oil line. Where the information related with the features in the maps drawing by geographic information system and by using special algorithms designed especially for GIS applications can put buffers around the features for specific distances in order to retrieve and find features within the area of the buffer. 


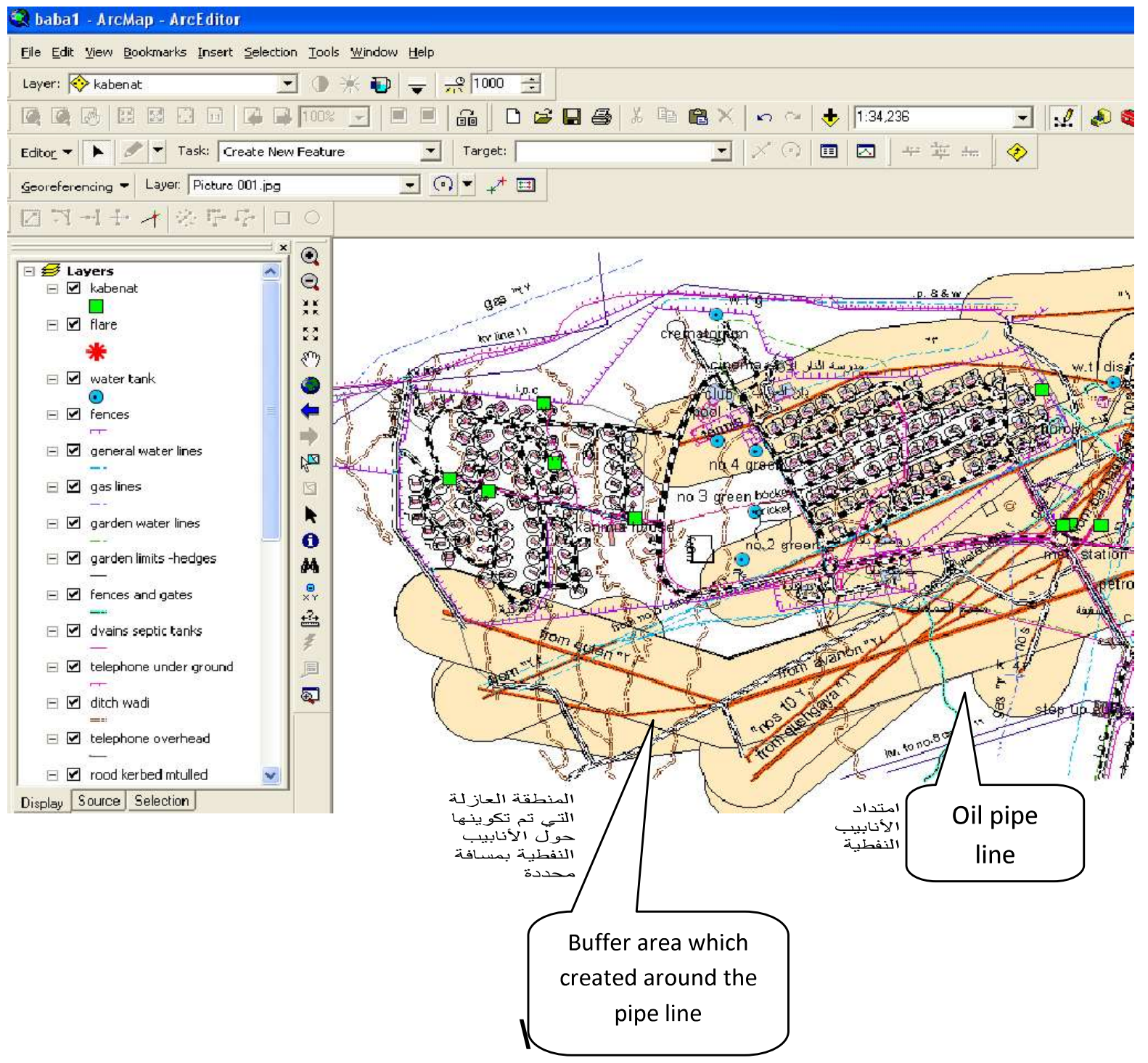

Fig. (6)

Figure (6) appears the creation of a buffer zone at a specified distance from the oil line along the tube. The located features within this area are in the danger zone. 


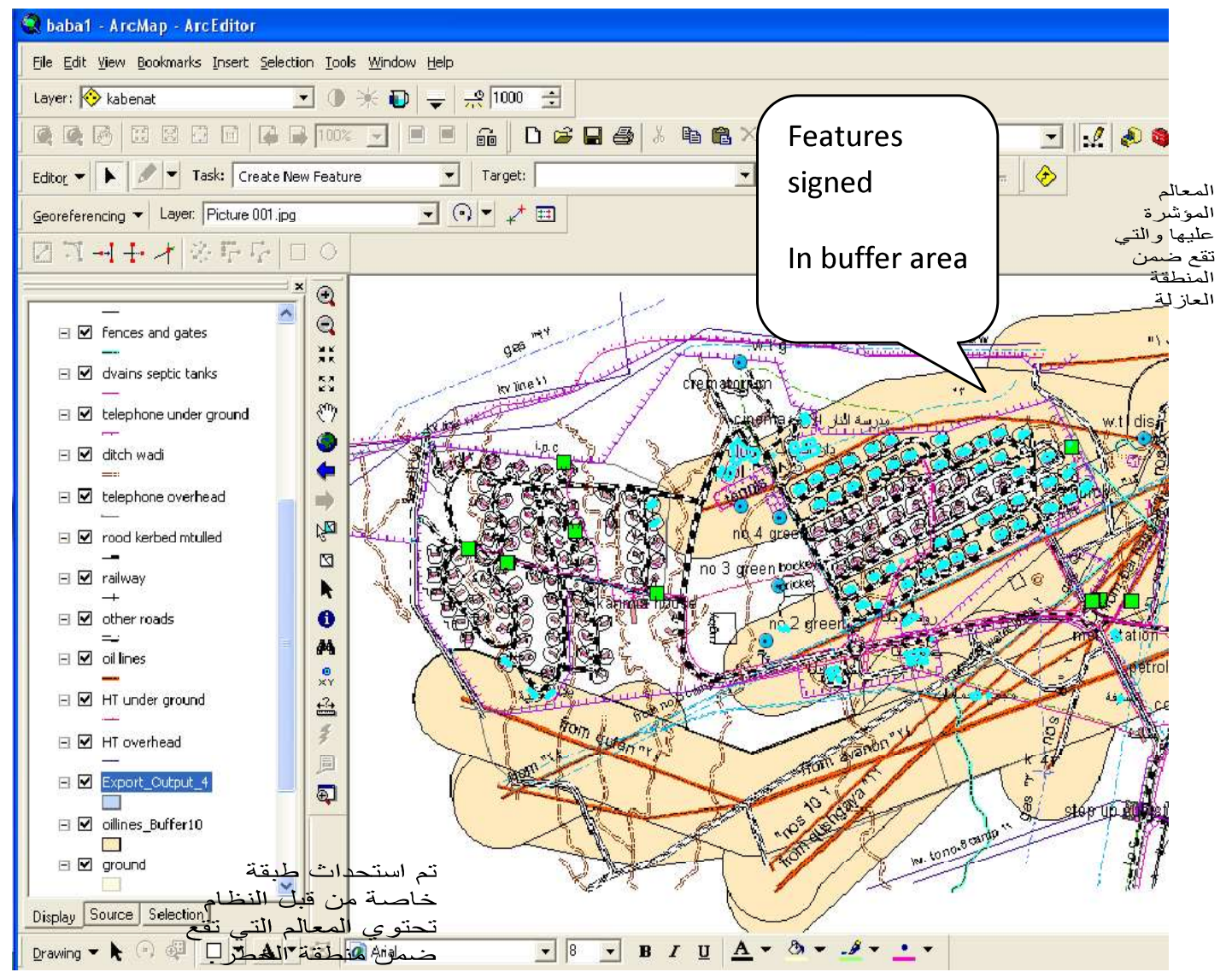

Fig. (7)

Figure (7) Appears designation of the buildings in the buffer zone and separated them in a new layer with their data, the system do that by a few steps, and marked them on the map by giving a new color different from the color in the original map. Building cursor in the figure (7) is located within the danger area and the system can provide the competent authorities by the list of numbers and locations of these buildings and quickly in case of any emergency. And it could be printed as a report that contains any information about the features as well as the required map. 


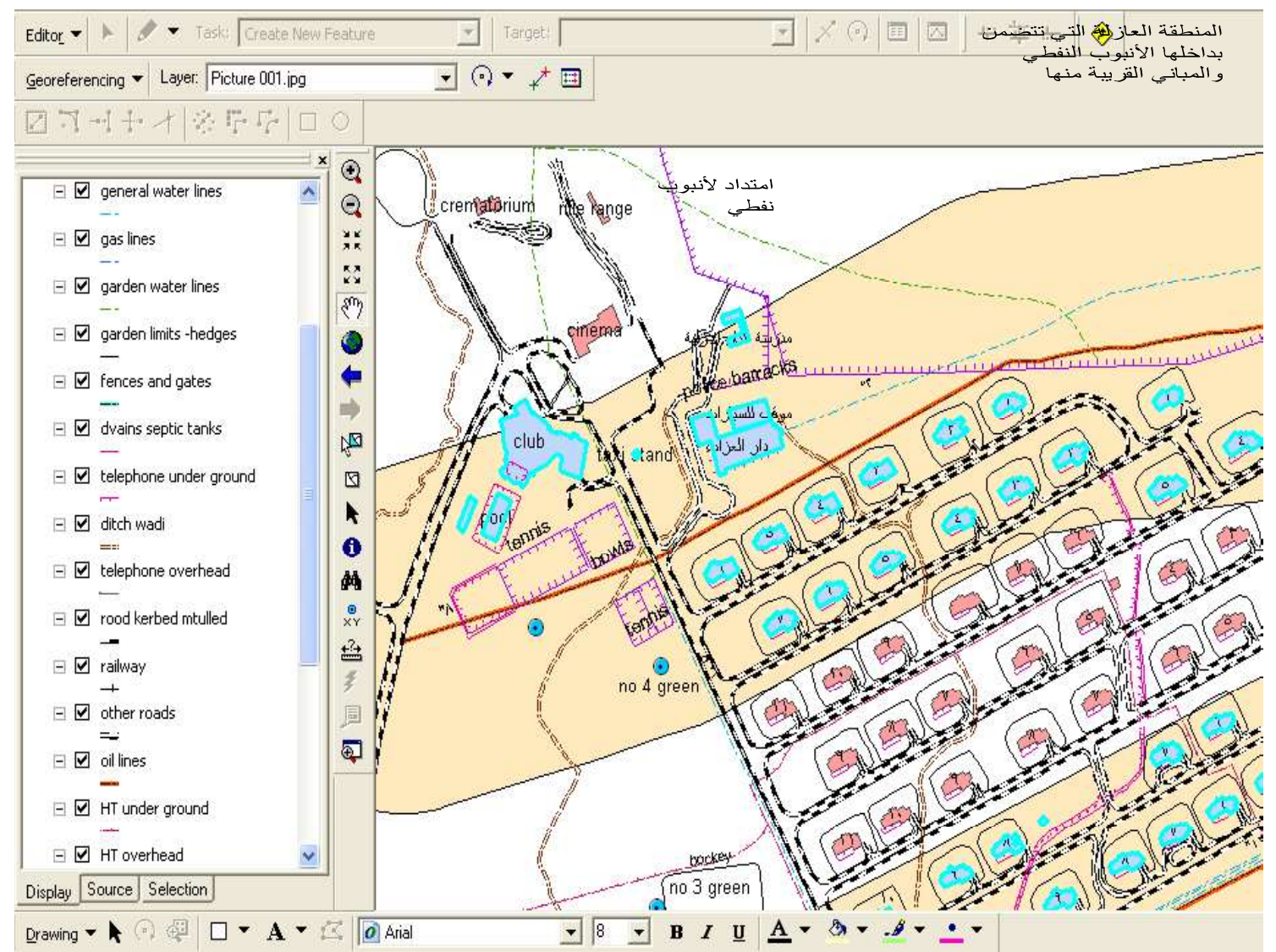

Fig. (8)

Figure (8) Shows enlarge part of the map.

\section{Application of geographic information system in industrial k1 area}

The organization and management of work in the industrial areas with existence a large number of reservoirs and complex networks of pipes and valves that control the process of receiving oil from the main lines and exporting or storing it in the oil reservoirs as needed requires scientifically and accurate dealing with this amount of spatial, descriptive data in these units, this particularity is found in geographic information systems. So to build a geographic database for this region we benefit from a satellite image of this area and paper maps which available and 


\section{NO.10 Journal of Petroleum Research \& Studies ( JPR \& S)}

by using program tools we can separated the features into multiple layers and draw every one $\mathrm{n}$ a layer such as reservoirs on a layer and oil pipelines in another layer, as well as the case of the valves and input the data for each features in its spreadsheet and every features represented on the map by the best geometric suited such as to represent oil pipelines we use a set of lines and represent valves as a set of points, reservoirs as spaces and therefore this manner permitted an easy dealings with the features on the map like addition, deletion, or concealment of some of the components of the map and to overcome the technical problems caused by processing large amounts of information in the same time. Whereas processed the problem in a layer better than processed it in the complete system and this is distinguishes digital maps and it is one of the GIS system characteristic. In addition to the deal with the data as layers with provide by analysis tools such as overlap and intersection between the layers lead to distinct production in analysis procedures and complex comparisons with the possibility of connecting several layers of spatial data and create maps that represent the results of that analysis.

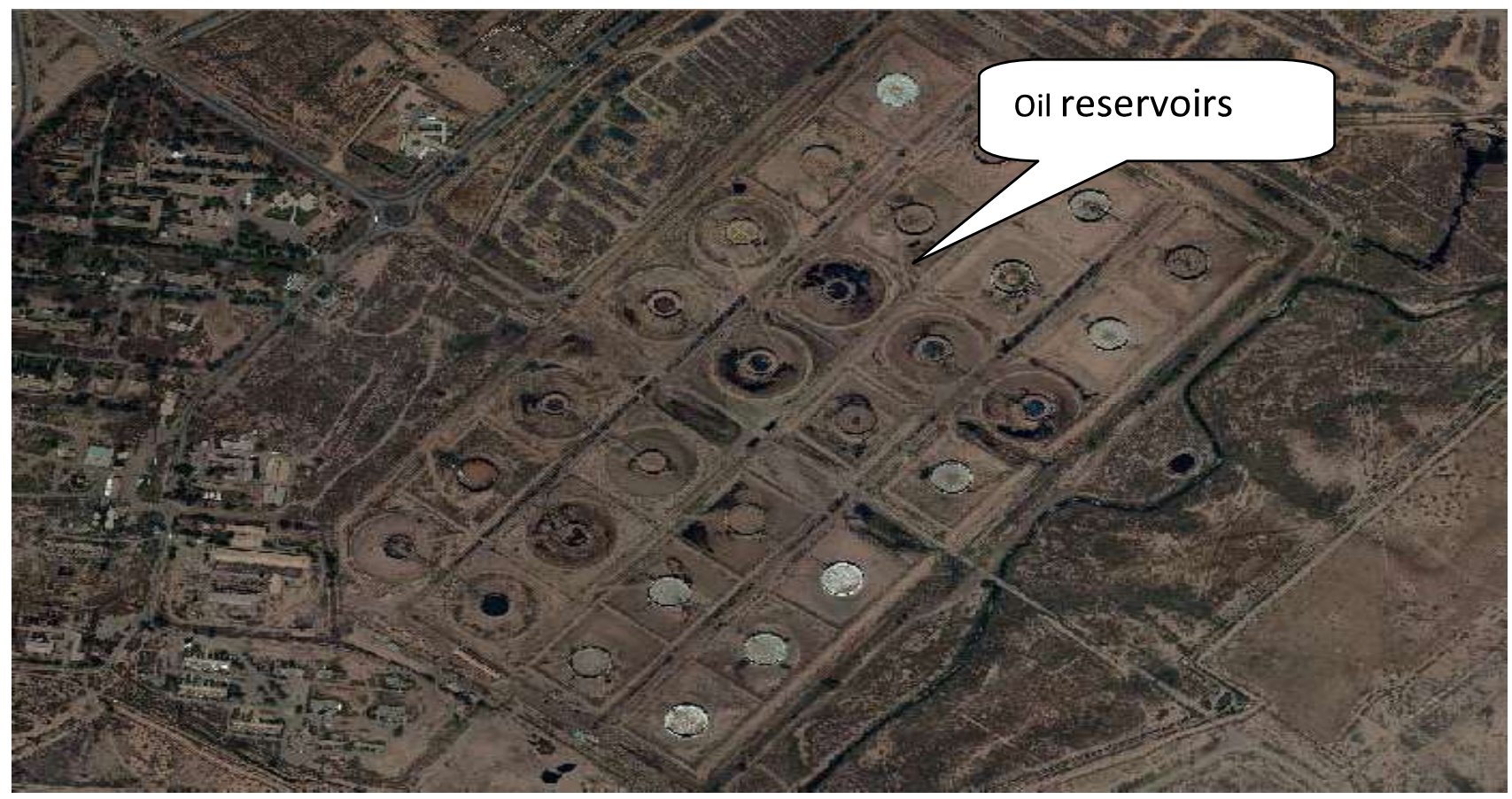

Fig. (9): Appears a satellite image for the industrial $k_{1}$ area 


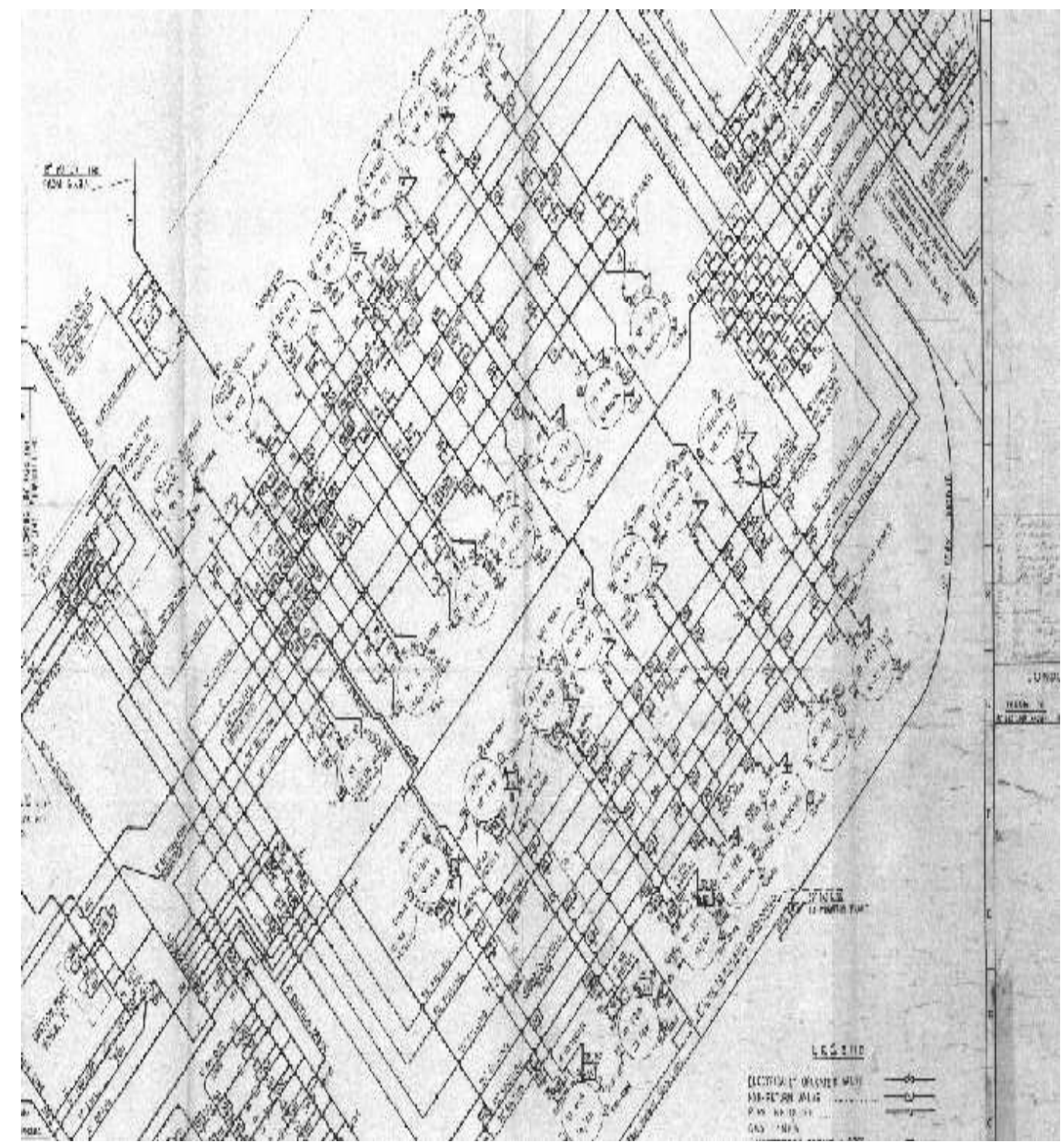

Fig.( 10)

Figure (10) represent the designe for the region which appear Oil reservoirs and oil pipelines and valve so we can use it as spatial information in geographic information system. 


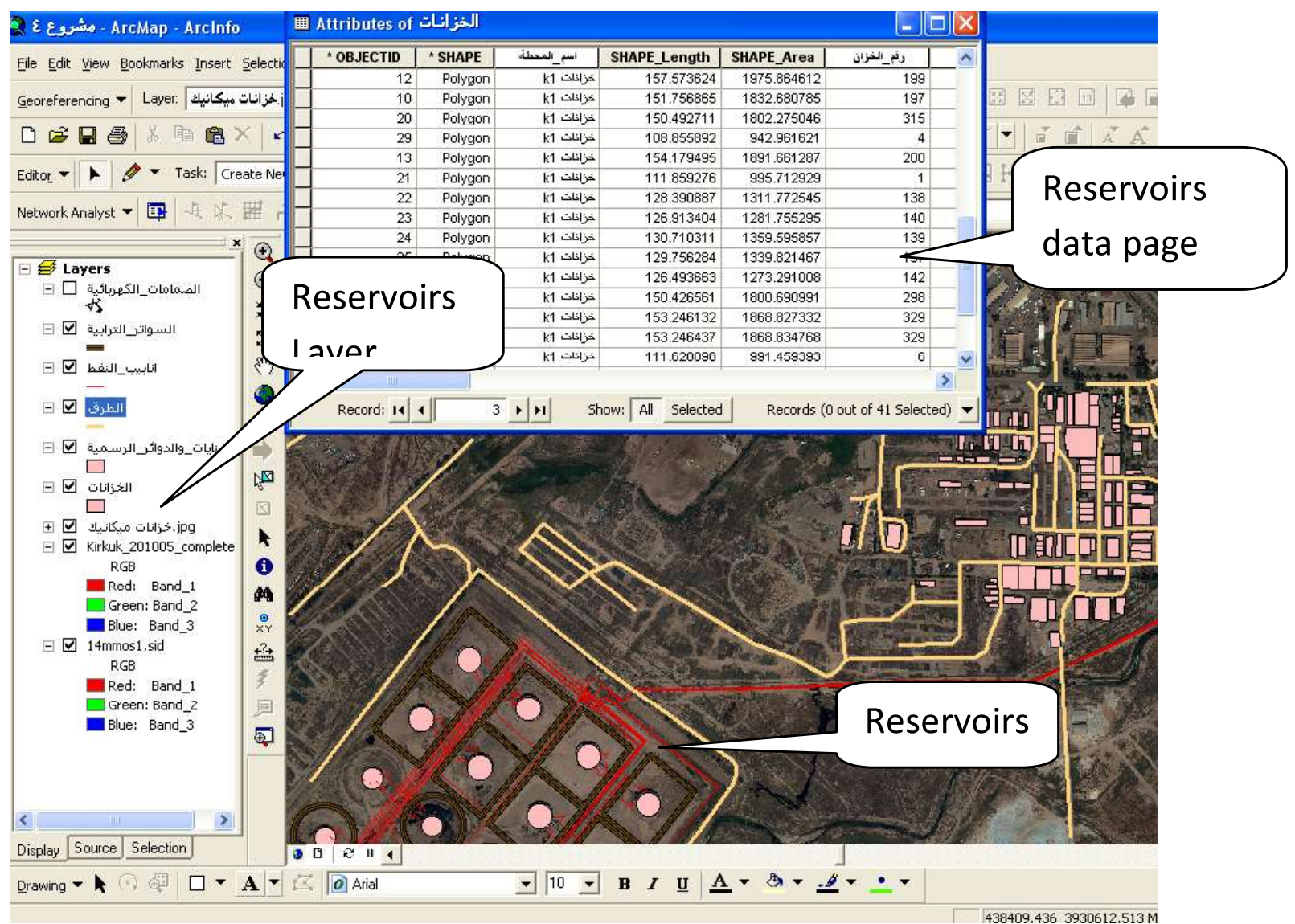

Fig. (11)

Figure (11) display dependence on a satellite image as basis for drawing the area that include tanks, oil pipelines and streets, buildings and the locations coordinate of these features are real and therefore the engineering measurements are identical to the reality, for example the length of the oil line specific in the map represents the same length on the ground and by the techniques application of the system like mathematical processors and technique of inquiry we can for example calculate the total lengths of pipes with a specific diameter so we can estimate the cost of replacement in the state of perform maintenance operations on them, as well as the availability of information for example the number of pumping stations, oil flow and the movement of oil and the end stations of the pipe and valves along the route of the line and by use of special system techniques we can pick the shortest path between two points and which is the most suitable for the transport of 
oil in the direction of the technical, economic and determine the number and location of stations.

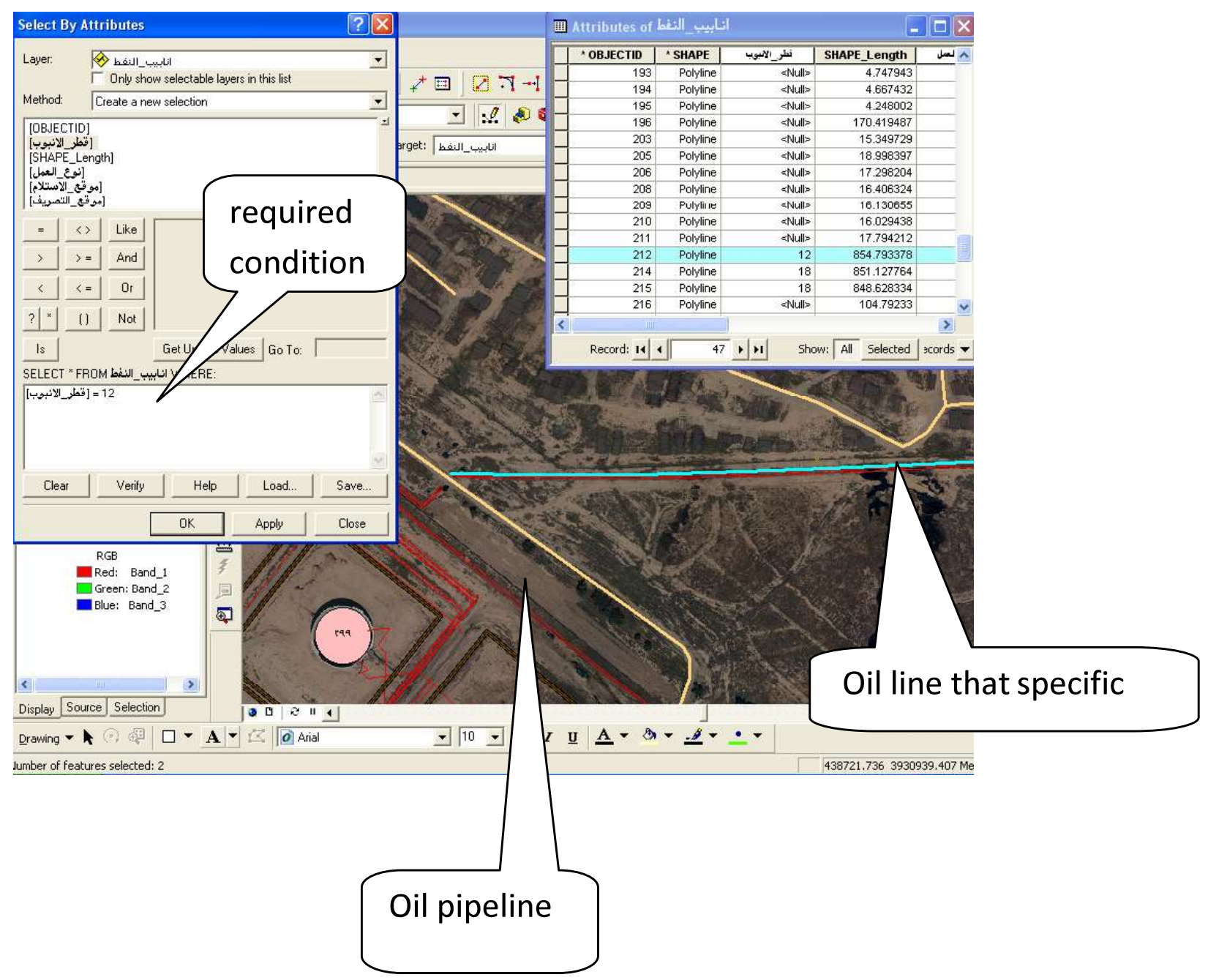

Fig. (12)

Figure (12) shows the inquiry property, in GIS system where we can search and selection dependence on available data in the data tables and show the importance of this property when dealing with large data where are querying on the data stored in tables or through the sites where it is the query process to find a features that proximity or from afar or intersection with other features. So within a of very few period we can find oil lines with specific diameter and these 


\section{NO.10 Journal of Petroleum Research \& Studies ( JPR \& S)}

lines are signaled on the map and in the data table and can query on features check more than one condition .

The existence of a number of spatial analysis tools such as overly and intersection between the layers according to certain conditions and query provides an exceptional opportunity to walk with accurate scientific steps to facilitate

Required tasks in the institutions for example when receipt the oil in storing stations and need to stored in the reservoirs we can choose the most appropriate reservoir for storage by querying on field for the level of oil in the reservoirs and thus we can reaching to the pipes and valves that must be employment to secure the oil movement to the required oil reservoirs. That is investigation to reach the right decisions in less time and more accuracy

\section{Conclusion}

Geographic information system (GIS) is an effective and distinct tool in the planning and management of projects where the information systems are for storing the information and processed it mathematically without the possibility of linking the information with its real location on the surface of the earth which is available in geographic information system but it is not easy to apply such modern systems completely because of the difficulty of providing scientific and material necessary potential to activate such systems which depends on the area of the region that the system applied on and required tasks as well as the high cost of programs and the need for fast and powerful hardware to run those programs. And add to that there are a few experienced people to manage such projects and the difficulty of get data as a result of damage the most paper maps which stored in poor conditions. Despite all the obstacles it is necessary to start working with this system in the institutions to avoidance enlargement the scientific gap with the 


\section{NO.10 Journal of Petroleum Research \& Studies ( JPR \& S)}

advanced countries in the field of technological information that has become apply this system to all areas where the institutions in advanced countries using geographic information systems to store and archives the thousands of maps and charts in a unified digital system, saving thereby huge amounts of money spent on manual and primitive labor files and lost maps. The other reason to implement this system is to try to save the remains of data and maps before damaged as a result of the use and bad storage. Also one of the major advantages of GIS is its ability to store and manage geographic information and connecting the different layers of data and analysis them, thus contribute in development of new strategies for the work and reaching the best solutions for problems in a little time. 


\section{References}

1. Geographic information system GIS / Practical Guide for the full system ARCVIEW9/ translation by Dr. Engineer Haitham yousef Zarkta.

2. Dr. Gomaa M. Dawod; "An introduction to the Digital maps".

3. Dr. Gomaa M. Dawod; "Principles of spatial analysis in geographic information system".

4. Geographic information system magazine, number 1

5. Dr. Badr Taha; Geographic messages, support decision industry and spatial analysis in geographic information system.

6. An introductory guide ARCGIS9.X program using the first level the number of graphic Information Systems Division, Department of Information, Technology, Ministry of Municipalities and Public Works. 